\title{
ZEUS-2D SIMULATIONS OF LASER-DRIVEN RADIATIVE SHOCK EXPERIMENTS
}

\author{
D.R. LEIBRANDT ${ }^{1}$, R.P. DRAKE ${ }^{1}$ and J.M. STONE ${ }^{2}$ \\ ${ }^{1}$ Department of Atmospheric, Oceanic, and Space Sciences, University of Michigan; \\ E-mail:dleibran@umich.edu \\ ${ }^{2}$ Department of Astrophysical Sciences, Princeton University
}

(Received 20 April 2004; accepted 1 June 2004)

\begin{abstract}
A series of experiments is underway using the Omega laser to examine radiative shocks of astrophysical relevance. In these experiments, the laser accelerates a thin layer of low- $Z$ material, which drives a strong shock into xenon gas. One-dimensional numerical simulations using the HYADES radiation hydrodynamics code predict that radiation cooling will cause the shocked xenon to collapse spatially, producing a thin layer of high density (i.e., a collapsed shock). Preliminary experimental results show a less opaque layer of shocked xenon than would be expected assuming that all the xenon accumulates in the layer and that the $\mathrm{X}$-ray source is a pure $\mathrm{K} \alpha$ source. However, neither of these assumptions is strictly correct. Here we explore whether radial mass and/or energy transport may be significant to the dynamics of the system. We report the results of two-dimensional numerical simulations using the ZEUS-2D astrophysical fluid dynamics code. Particular attention is given to the simulation method.
\end{abstract}

Keywords: radiation hydrodynamics, methods: numerical

\section{Introduction}

This work uses the ZEUS-2D astrophysical fluid dynamics code (Stone and Norman, 1992) with a flux-limited diffusion radiation module (Turner and Stone, 2001) to model a set of laser-driven radiative shock experiments. While several target designs have been used in experiments, this work assumes the target design shown schematically in Figure 1. The target is cylindrically symmetric. The drive disk is $72 \mu \mathrm{m}$ thick polystyrene with a density of $1.05 \mathrm{~g} / \mathrm{cm}^{3}$. The tube is $4 \mathrm{~mm}$ long, has an inner diameter of $600 \mu \mathrm{m}$ and an outer diameter of $800 \mu \mathrm{m}$, and is also $1.05 \mathrm{~g} / \mathrm{cm}^{3}$ polystyrene. The xenon gas has a density of $0.006 \mathrm{~g} / \mathrm{cm}^{3}$. The target is driven by a $1 \mathrm{~ns}$ full width at half maximum laser pulse with an irradiance of $1.7 \times 10^{15} \mathrm{~W} / \mathrm{cm}^{2}$ at the center of the drive disk. The irradiance falls off by $45 \%$ at the outer diameter of the tube. As ZEUS-2D does not have a laser module, the initial conditions are linearly interpolated from a set of one-dimensional axial simulations of different radial coordinates at $1 \mathrm{~ns}$ (i.e., immediately after the drive laser turns off) generated by HYADES. HYADES is a one-dimensional, Lagrangian, single-fluid, three-temperature code with multigroup flux-limited diffusion radiation transport 


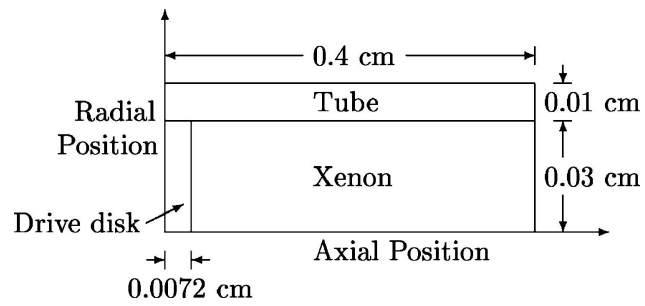

Figure 1. Target schematic. The drive disk and tube are $1.05 \mathrm{~g} / \mathrm{cm}^{3}$ polystyrene and the xenon is $0.006 \mathrm{~g} / \mathrm{cm}^{3}$. The target is driven from the left by a $1 \mathrm{~ns}$ laser pulse with an average irradiance of $1.4 \times 10^{15} \mathrm{~W} / \mathrm{cm}^{2}$.

(Larsen and Lane, 1994). The HYADES laser irradiance profile is scaled to $25 \%$ of the experimental irradiance to account for lateral heat transport, which is determined by matching simulated and experimental interface and shock motions in previous experiments. The heating of the xenon gas by radiation from the shock is so large that preheat by suprathermal electrons is unlikely to affect the dynamics.

\section{Simulation Method}

ZEUS-2D is a two-dimensional, Eulerian, single-fluid, two-temperature code with gray radiation transport (Stone and Norman, 1992; Stone et al., 1992). We replaced the original discrete ordinate radiation module with a flux-limited diffusion radiation module based on the work of Turner and Stone (2001) for computational efficiency. The equations solved are

$$
\begin{aligned}
\frac{D \rho}{D t}+\rho \nabla \cdot \mathbf{v} & =0, \\
\rho \frac{D \mathbf{v}}{D t} & =-\nabla p+\frac{1}{c} \rho \kappa_{\mathrm{R}} \mathbf{F}, \\
\rho \frac{D}{D t}\left(\frac{E}{\rho}\right) & =-\nabla \cdot \mathbf{F}-\nabla \mathbf{v}: \mathbf{P}+4 \pi \rho \kappa_{\mathrm{P}} B-c \rho \kappa_{\mathrm{P}} E,
\end{aligned}
$$

and

$$
\rho \frac{D}{D t}\left(\frac{e}{\rho}\right)=-p \nabla \cdot \mathbf{v}-4 \pi \rho \kappa_{\mathrm{P}} B+c \rho \kappa_{\mathrm{P}} E
$$

where $D / D t \equiv \partial / \partial t+\mathbf{v} \cdot \nabla$ is the convective derivative; $\rho, e, \mathbf{v}$, and $p$ are the material mass density, energy density, velocity, and pressure; $E, \mathbf{F}$, and $\mathbf{P}$ are the radiation energy density, flux, and pressure tensor; $\kappa_{\mathrm{R}}$ and $\kappa_{\mathrm{P}}$ are the Rosseland and Planck mean specific opacities; $B$ is the Planck function; $c$ is the speed of light; and $t$ is time. Note that this work uses the Rosseland mean specific opacity for 
the radiation flux terms and the Planck mean specific opacity for the heating and cooling terms. These equations are closed by the addition of an equation of state, constitutive relations for the Planck function and opacities, and a flux limiter.

This work uses the ideal gas equation of state $p=(\gamma-1) e$ where $\gamma$ is the ratio of specific heats. $\gamma=5 / 3$ for polystyrene and $11 / 9$ for xenon. The latter is determined by the strong shock jump condition, $\rho_{2} / \rho_{1}=(\gamma+1) /(\gamma-1)$, using densities from a HYADES simulation with radiation transport suppressed. The Planck function $B=(\sigma / \pi) T^{4}$ is computed using the implicitly defined material temperature $T=$ $(\gamma-1) \mu e /(\Re \rho(1+Z))$ with an average ionization $Z=20 \sqrt{T_{k e V}}$ (Drake, in preparation) where $\sigma$ is the Stefan-Boltzmann constant, $\mathscr{R}$ is the gas constant, $\mu$ is the dimensionless mean particle mass, and $T_{k e V}$ is the material temperature in $\mathrm{keV}$. $\mu=6.5$ for polystyrene and 131.3 for xenon. The opacities used are least squares fits to the LANL SESAME tables \#17593 (polystyrene) and \#15190 (xenon). The (Minerbo, 1978) flux limiter is used for this work, but there is little difference when the (Levermore and Pomraning, 1981) flux limiter is used instead.

It is found that the alternating direction-implicit (ADI) matrix solver used to evolve the radiation flux divergence term does not converge when the optical depth per zone is too small; these simulations have an optical depth per zone of order 0.1 in the xenon region. This work replaces the ADI matrix solver with a band matrix lower upper factorization matrix solver from the LAPACK numerical linear algebra package (Anderson et al., 1999).

\section{Results}

Figure 2 shows the simulation results. Note that the detailed structure of the polystyrene/xenon interface, including the peculiar behavior on axis, is the unphysical result of using a two-dimensional code to model three-dimensional

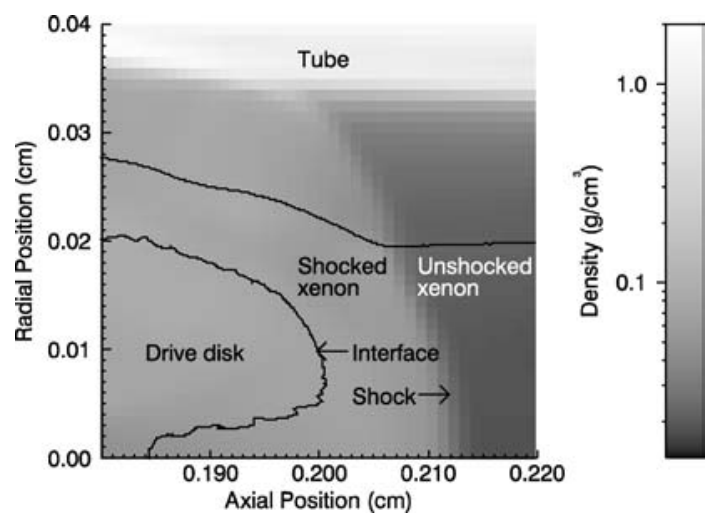

Figure 2. ZEUS-2D simulation results. The density is plotted in false color as a function of position at $20 \mathrm{~ns}$. 
hydrodynamic instabilities. There is a significant accumulation of shocked xenon between the drive disk and the tube. In addition, the shock has a large curvature. These results suggest a mechanism for shocked xenon loss that might explain the low opacity of the shocked xenon layer.

\section{Conclusions}

This work reports the results of ZEUS-2D simulations that suggest an explanation of the low opacity of the shocked xenon layer. Future directions include validation and quantitative comparison with experiment.

\section{Acknowledgements}

This work is supported at the University of Michigan by the U.S. Department of Energy under grants DE-FG03-99DP00284, DE-FG03-00SF22021, and other grants and contracts.

\section{References}

Anderson, E., Bai, Z., Bischof, C., Blackford, S., Demmel, J., Dongarra, J., Du Croz, J., Greenbaum, A., Hammarling, S., McKenney, A. and Sorensen, D.: 1999, LAPACK Users' Guide, SIAM, Philadelphia, PA.

Drake, R.P.: in preparation, High Energy Density Physics: From Inertial Fusion to Experimental Astrophysics, Springer-Verlag, New York, NY.

Larsen, J.T. and Lane, S.M.: 1994, J. Quant. Spectrosc. Radiat. Transfer 51, 179.

Levermore, C.D. and Pomraning, G.C.: 1981, ApJ 248, 321.

Minerbo, G.N.: 1978, J. Quant. Spectrosc. Radiat. Transfer 31, 149.

Stone, J.M., Mihalas, D. and Norman, M.L.: 1992, ApJS 80, 819.

Stone, J.M. and Norman, M.L.: 1992, ApJS 80, 753.

Turner, N.J. and Stone, J.M.: 2001, ApJS 135, 95. 